\title{
Scattering Parameters Characterization of Periodically-Nonuniform Transmission Lines with a Perturbative Technique
}

\author{
Xiaokang Liu, Xinglong Wu, Student Member, IEEE, Flavia Grassi, Senior Member, IEEE, Giordano \\ Spadacini, Senior Member, IEEE, Dries Vande Ginste, Senior Member, IEEE, and Sergio A. Pignari, Fellow, IEEE
}

\begin{abstract}
In this manuscript, a novel procedure for the frequency-domain solution of nonuniform transmission lines (NUTLs) is presented. The procedure is based on a recentlyproposed perturbative technique, which is proven to be computationally more efficient than standard solution approaches, which are based on line subdivision into uniform cascaded sections (UCS). With respect to the original perturbation technique, the procedure proposed here offers more flexibility, as it provides a representation of the NUTL under analysis in terms of $S$ - and/or $T$-parameters at its ports. Moreover, it retains the same prediction accuracy at the price of a slight increase in computational burden, which can be mitigated anyway through parallel-computing. Furthermore, even without ad hoc (parallel) implementations, the proposed procedure outperforms other approaches to solve differential lines with partially- or fullyrepetitive geometries. Namely, it assures accurate prediction of output quantities with reduced simulation time. This is proven by three application examples, namely two differential trapezoidal tabbed lines (one with interdigital tabs and one with facing tabs) and a differential microstrip line with a varying common-mode $(\mathrm{CM})$ impedance (as such reducing $\mathrm{CM}$ noise). Comparison with full-wave simulations allows assessing the prediction accuracy of the proposed procedure. Comparison with the aforementioned transmission-line based solutions allows appreciating the enhanced computational efficiency.
\end{abstract}

Index Terms-Tabbed lines, scattering parameters, scattering transfer parameters, nonuniform transmission lines, perturbative technique.

\section{INTRODUCTION}

In practical engineering problems, many wiring and printed circuit board (PCB) structures can be modeled as nonuniform transmission lines (NUTLs), where line non-uniformity might be either desired (e.g., in twisted-wire pairs or advanced trace-routing schemes for high-speed signal transmission) or undesired (e.g., due to imperfections in the manufacturing process). The standard solution approach, also known as the Uniform Cascaded Section (UCS) method, subdivides the line into sufficiently short sub-sections with nearly constant crosssection. The corresponding chain-parameter matrices [1] or $\mathrm{ABCD}$ matrices [2] are then cascaded in order to evaluate

Manuscript received October 7, 2019, revised January 7, 2020.

X. Liu, X. Wu, F. Grassi, G. Spadacini, and S. A. Pignari are with the Department of Electronics, Information and Bioengineering, Politecnico di Milano, 20133 Milan, Italy, e-mail: xiaokang.liu@polimi.it; xinglong.wu@polimi.it; flavia.grassi@polimi.it; giordano.spadacini@polimi.it; sergio.pignari@polimi.it.

D. Vande Ginste is with the IDLab/Electromagnetics Group, Department of Information Technology, Ghent University/imec, 9052 Gent, Belgium (e-mail: dries.vandeginste@ugent.be). voltages and currents at line terminals. To mitigate the computational burden of the aforesaid approach, which may involve a very fine discretization in case of strong line non-uniformity, an alternative technique was recently proposed in [3], [4]. This novel technique treats line non-uniformity as a perturbation of an equivalent uniform transmission line (TL). Accordingly, NUTL equations are recast and solved as the equations pertaining to an equivalent uniform TL driven by distributed sources. These sources depend on the difference between the actual p.u.l. parameters of NUTL under analysis and those of the equivalent uniform TL, and as such accounting for the line nonuniformity. They are iteratively updated in order to achieve an accurate prediction of the voltages and currents at the line terminals. Several application examples [4]-[7] proved the computational effectiveness of this technique with respect to the traditional UCS approach, yet retaining the same prediction accuracy.

In spite of undoubted advantages in terms of computational time, the approach presented in [3]-[7] results to be less flexible than the UCS method, since output quantities of the iterative procedure are the voltages and currents evaluated for a specific set of terminal conditions. Conversely, the UCS method provides a network representation at the NUTL's ports, which can be readily used in combination with different terminal conditions and/or subsequently cascaded with other lumped or distributed networks.

To overcome the aforesaid limitation, an alternative implementation of the perturbation technique is proposed in this manuscript, which resorts to an S-parameter representation of the NUTL under analysis. In this novel approach, the $(n+1)$ NUTL under analysis is characterized as a $2 n$-port network, whose $S$-parameters are evaluated by applying the perturbation technique in combination with specific terminal conditions enforced at the NUTL's ports. With respect to the original implementation, the slight disadvantage of the proposed approach stems from the need to solve the line $2 n$ times. However, exploiting repetitive geometrical properties or invoking parallel computing can alleviate this issue.

Moreover, the novel approach results to be particularly suitable and efficient to model differential line structures characterized by fully or partially-repetitive geometries. Such advanced trace routing schemes were recently introduced for high-speed data transmission with reduced interference, e.g., [8]-[15]. Indeed, in these cases, the basic cells (building blocks) constituting the periodic structure are characterized. 
This yields a fast evaluation procedure, as, e.g., for differential lines this requires the solution of $2 n=4$ sub-problems only and the cascading merely involves the multiplication of the building block's pertinent $T$-parameter matrices.

In the manuscript, specific application examples are presented, with the objective to prove the accuracy and computational efficiency of the proposed procedure. First, two differential trapezoidal tabbed-lines (with interleaved and facing tabs), used for high-speed data transmission with limited crosstalk [10], [11], are considered. Next, a novel differential microstrip geometry specifically designed [12], [13] to suppress commonmode $(\mathrm{CM})$ noise in specific frequency intervals is analyzed. For all three structures, the advocated approach is compared to full-wave simulations to assess the validity of the assumptions of TL theory in the presence of line nonuniformities. Moreover, the computational time required by the proposed procedure is compared to other TL-based methods (i.e., the UCS method and the original implementation of the perturbation technique), as such demonstrating equal prediction accuracy in less computational time.

The remainder of this manuscript is organized as follows. In Section II, the basic principles of the perturbation technique in [4] are briefly revised. In Section III, the proposed procedure is presented, yielding $S$ - and $T$-parameters representation (and characterization) of the NUTL under analysis. Accuracy and computational efficiency are discussed in Sections IV and $\mathrm{V}$, where the proposed procedure is exploited to solve two differential trapezoidal tabbed-lines and one differential microstrip with CM suppression, respectively. Conclusions are drawn in Section VI.

\section{Basic Principles of the Perturbation Technique}

In this section, the basic principles of the perturbation technique recently proposed in [3], [4] for the efficient solution of NUTLs are briefly recalled. Particularly, the need for directly enforcing the terminal constraints is stressed, since it represents the most significant limitation of the technique, whereas in this work a reformulation is proposed to overcome this limitation.

Consider an $(n+1)$-conductor NUTL. The basic idea behind the method in [3], [4] is to express the place-dependent $n \times n$ p.u.l. impedance and admittance matrices $\mathbf{Z}(z), \mathbf{Y}(z)$ appearing in the frequency-domain phasor equations, i.e.,

$$
\begin{aligned}
\frac{d}{d z} \mathbf{V}(z) & =-\mathbf{Z}(z) \mathbf{I}(z) \\
\frac{d}{d z} \mathbf{I}(z) & =-\mathbf{Y}(z) \mathbf{V}(z)
\end{aligned}
$$

as the superposition of two matrices:

$$
\begin{aligned}
\mathbf{Z}(z) & =\overline{\mathbf{Z}}+\Delta \mathbf{Z}(z) \\
\mathbf{Y}(z) & =\overline{\mathbf{Y}}+\Delta \mathbf{Y}(z)
\end{aligned}
$$

where $z$ denotes the longitudinal coordinate along the TL, and $\mathbf{V}(z), \mathbf{I}(z)$ are $n \times 1$ vectors collecting the $n$ line voltages $V_{i}(z)$ and currents $I_{i}(z)$ in phasor form $(i=1, \ldots, n)$, respectively. In (2), the place-independent matrices $\overline{\mathbf{Z}}=\frac{1}{l} \int_{0}^{l} \mathbf{Z}(z) d z$ and
$\overline{\mathbf{Y}}=\frac{1}{l} \int_{0}^{l} \mathbf{Y}(z) d z$ are obtained by averaging the actual p.u.l. parameters along the line length, and can be therefore interpreted as the p.u.l. parameters of a TL with constant cross-section (uniform TL). Conversely, the entries of matrices $\Delta \mathbf{Z}(z)$ and $\Delta \mathbf{Y}(z)$ are still place-dependent, and account for the deviation (perturbation) of the actual p.u.l. parameters from their average value.

In a similar fashion, by expressing voltages and currents as the superposition of an average contribution $\mathbf{V}_{0}, \mathbf{I}_{0}$ and a suitable number of perturbation terms $\mathbf{V}_{k}, \mathbf{I}_{k}$, i.e.,

$$
\begin{gathered}
\mathbf{V}(z)=\mathbf{V}_{0}(z)+\sum_{k=1}^{K} \mathbf{V}_{k}(z) \\
\mathbf{I}(z)=\mathbf{I}_{0}(z)+\sum_{k=1}^{K} \mathbf{I}_{k}(z)
\end{gathered}
$$

the line solution is iteratively achieved as follows. First, the average $\left(0^{\text {th }}\right.$-order) solution is obtained by combining the equations pertinent to the average-uniform line

$$
\begin{aligned}
& \frac{d}{d z} \mathbf{V}_{0}(z)+j \omega \overline{\mathbf{Z}} \mathbf{I}_{0}(z)=0 \\
& \frac{d}{d z} \mathbf{I}_{0}(z)+j \omega \overline{\mathbf{Y}} \mathbf{V}_{0}(z)=0
\end{aligned}
$$

with the constraints imposed by the terminal networks. Then, the perturbation terms are evaluated by including the effects due to line non-uniformity. This requires the iterative solution of the following set of equations:

$$
\begin{aligned}
\frac{d}{d z} \mathbf{V}_{k}(z)+\overline{\mathbf{Z}} \mathbf{I}_{k}(z) & =-\boldsymbol{\Delta} \mathbf{Z}(z) \mathbf{I}_{k-1}(z) \\
\frac{d}{d z} \mathbf{I}_{k}(z)+\overline{\mathbf{Y}} \mathbf{V}_{k}(z) & =-\boldsymbol{\Delta} \mathbf{Y}(z) \mathbf{V}_{k-1}(z)
\end{aligned}
$$

where the distributed sources, i.e., the terms on the right-hand side of (5), at the $k$-th step, depend on the perturbation entries in (5) through voltages and currents evaluated at step $k-1$. At each iteration, the line solution of (5) is achieved by enforcing the terminal constraints and by integrating the distributed sources along the line length. The procedure is iterated until the solution converges below a predefined threshold $\delta_{t h}$.

The technique is comparable with the traditional UCS approach (which discretizes the line into uniform sections [4]) in terms of prediction accuracy, yet offering significant advantages in terms of computational effort [4]-[6]. However, since terminal constraints have to be enforced at each iteration step: (a) the line solution must be re-evaluated whenever the terminal conditions change; and (b) the method is not suitable to cascade multiple TL sections. This last issue becomes especially relevant if the TL under analysis exhibits discontinuities (e.g., due to the presence of bends or connectors) or periodic non-uniformities (as in the examples shown in the remainder of this work).

\section{Proposed Solution Technique}

\section{A. Problem Statement and Objective}

The main limitation of the perturbation technique, briefly described in the previous section, is to be ascribed to the fact 
that, unlike standard solution approaches, it does represent the NUTL as a $2 n$-port network, but it provides the line solution directly in terms of voltages and currents at its terminals. Hence, it does not allow cascading the NUTL with other lumped or distributed sections. To overcome this limitation, the technique proposed here makes use of the original perturbation technique, in combination with a set of standard loads, with the objective to provide a $2 n$-port network description of the NUTL, yet retaining the advantages in terms of computational efficiency of the original perturbation technique.

In principle, any $2 n$-port representation can be adopted for this task. However, preliminary investigations (not detailed here for brevity) revealed that traditional representations based on impedance/admittance or chain-parameter matrices may lead to numerical instabilities of the obtained matrix entries, due to the strong reflections introduced at high frequencies by enforcing short/open-circuit conditions at the terminal ports. This limitation is mitigated by resorting to the $S$-parameter representation, since a reference impedance in the range of the NUTL's characteristic impedance can be used to terminate all ports. Once the S-parameters representation of the NUTL is available, it can be readily cascaded (through $T$-parameters) with other $2 n$ ports, such as connectors and/or other lumped or distributed sections (e.g., bent discontinuities or different TL sections), that were also characterized in terms of S-parameters (e.g. through Vector Network Analyzer (VNA) measurements or full-wave simulations).

\section{B. S-Parameter Characterization of the NUTL under Analysis}

Consider the generic $(n+1)$-conductor NUTL shown in Fig. 1. The relationships between the $2 n$ reflected waves ( $n$ at the left side, i.e., $b_{L, i}$, and $n$ at the right side, i.e., $\left.b_{R, i}\right)$ and the $2 n$ forward waves ( $n$ at the left side, i.e., $a_{L, j}$, and $n$ at the right side, i.e., $a_{R, j}$ ) at the $2 n$ ports are cast as

$\left[\begin{array}{c}b_{L, 1} \\ \vdots \\ b_{L, n} \\ b_{R, 1} \\ \vdots \\ b_{R, n}\end{array}\right]=\left[\begin{array}{cccccc}S_{1,1} & \cdots & S_{1, n} & S_{1, n+1} & \cdots & S_{1,2 n} \\ \vdots & \ddots & \vdots & \vdots & \ddots & \vdots \\ S_{n, 1} & \cdots & S_{n, n} & S_{n, n+1} & \cdots & S_{n, 2 n} \\ S_{n+1,1} & \cdots & S_{n+1, n} & S_{n+1, n+1} & \cdots & S_{n+1,2 n} \\ \vdots & \ddots & \vdots & \vdots & \ddots & \vdots \\ S_{2 n, 1} & \cdots & S_{2 n, n} & S_{2 n, n+1} & \cdots & S_{2 n, 2 n}\end{array}\right]\left[\begin{array}{c}a_{L, 1} \\ \vdots \\ a_{L, n} \\ a_{R, 1} \\ \vdots \\ a_{R, n} \\ (6)\end{array}\right]$

which in compact form reads

$$
\left[\begin{array}{l}
\mathbf{b}_{\mathrm{L}} \\
\mathbf{b}_{\mathrm{R}}
\end{array}\right]=\left[\begin{array}{ll}
\mathbf{S}_{\mathrm{L}, \mathrm{L}} & \mathbf{S}_{\mathrm{L}, \mathrm{R}} \\
\mathbf{S}_{\mathrm{R}, \mathrm{L}} & \mathbf{S}_{\mathrm{R}, \mathrm{L}}
\end{array}\right]\left[\begin{array}{l}
\mathbf{a}_{\mathrm{L}} \\
\mathbf{a}_{\mathrm{R}}
\end{array}\right]
$$

where subscripts $L$ and $R$ denote the left and right side of the NUTL under analysis, respectively.

The matrix entries are evaluated column by column, by imposing an excitation incident wave at one port at the time, the other ports being loaded with the reference impedance $Z_{C}$, and by evaluating the resulting reflected waves at all ports. For instance, with reference to the specific test case considered in Fig. 1 (where the only non-null forward wave is $a_{L, j}$ ), the entries of the $j$-th column of the $S$-parameter matrix in (6) are obtained as

$$
S_{i, j}=\left.\frac{b_{L(R), i}}{a_{L, j}}\right|_{a_{L, k}=0, k \neq j ; a_{R, m}=0, m=1 \ldots n}
$$

and similarly for the entries of all the other columns.

In terms of voltages and currents (see Fig. 1), enforcing a unitary forward wave $a_{j}$ at port $j$, viz.

$$
a_{j}=\frac{1}{2 \sqrt{Z_{C}}}\left(V_{j}+Z_{C} I_{j}\right)=\frac{V_{S}}{2 \sqrt{Z_{C}}}=1
$$

is equivalent to connecting to this port to a non-ideal voltage source with internal impedance $Z_{C}$ and open-circuit voltage

$$
V_{S}=2 \sqrt{Z_{C}}
$$

The resulting reflected waves at all $2 n$ ports are calculated by the perturbation technique described in Section II, as

$$
b_{L(R), i}=\frac{1}{2}\left[\frac{V_{L(R), i}}{\sqrt{Z_{C}}}-\sqrt{Z_{C}} I_{L(R), i}\right]
$$

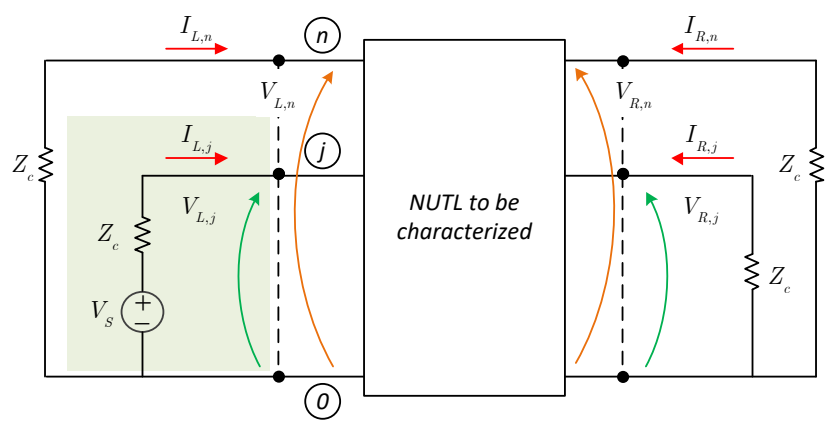

Fig. 1. Terminal conditions for the evaluation of the $j$-th column entries of the $S$-parameter matrix in (6).

yielding the entries of the $j$-th column in (6). The evaluation has to be repeated $2 n$ times, in order to estimate all the entries in (6). It is worth mentioning that this does not necessarily entails that the required computational time is multiplied by $2 n$, since the aforementioned $2 n$ sub-problems are characterized by the very same set of TL equations and only the terminal conditions change from one sub-problem to the other. Moreover, distinctive repetitive geometrical features of the structure under analysis can be often exploited and, furthermore, the procedure can significantly benefit from parallel computing.

With respect to the traditional formulation of the perturbation technique, the proposed procedure offers more flexibility, since the obtained $S$-parameter representation can be readily re-used in combination with different sets of terminal conditions, and can be cascaded with other line sections, connectors, bend discontinuities and so on. In particular, the proposed technique definitely outperforms the performance of the traditional perturbation technique for the analysis of NUTLs exhibiting periodic geometries. For instance, this is the case for ideal twisted wire pairs but also for new classes of periodic PCB traces, as the application examples presented in the following will illustrate. 


\section{Cascading and Prediction of Terminal Voltages and Cur- rents of the Entire NUTL}

Once the $S$-parameter representation (7) is available, it is readily converted into a $T$-parameter description [16]:

$$
\left[\begin{array}{l}
\mathbf{a}_{\mathrm{L}} \\
\mathbf{b}_{\mathrm{L}}
\end{array}\right]=\left[\begin{array}{cc}
\mathbf{T}_{\mathrm{L}, \mathrm{L}} & \mathbf{T}_{\mathrm{L}, \mathrm{R}} \\
\mathbf{T}_{\mathrm{R}, \mathrm{L}} & \mathbf{T}_{\mathrm{R}, \mathrm{R}}
\end{array}\right]\left[\begin{array}{l}
\mathbf{b}_{\mathrm{R}} \\
\mathbf{a}_{\mathrm{R}}
\end{array}\right]
$$

where the $T$-parameter entries can be obtained as in [17] so that different line sections can be cascaded by simply multiplying the corresponding $T$-parameter matrices. This is illustrated in Fig. 2 for two sections.

Without loss of generality, the cascaded TL network of Fig. 2 is now further analyzed. Port constraints are incorporated by adopting an equivalent Thevenin representation of the terminal networks at both ends $z=0$ and $z=L$, i.e., $\mathbf{V}(0)=\mathbf{V}_{\mathbf{S}}-\mathbf{Z}_{\mathbf{S}} \mathbf{I}(0)$ and $\mathbf{V}(L)=\mathbf{V}_{\mathbf{L}}+\mathbf{Z}_{\mathbf{L}} \mathbf{I}(L)$, where $\mathbf{V}_{\mathbf{S}}$ and $\mathbf{V}_{\mathrm{L}}$ denote the $n \times 1$ generalized Thevenin voltage source vectors at the left and right end, respectively, and $\mathbf{Z}_{\mathrm{S}}$ and $\mathbf{Z}_{\mathrm{L}}$ are the corresponding $n \times n$ generalized Thevenin impedance matrices.

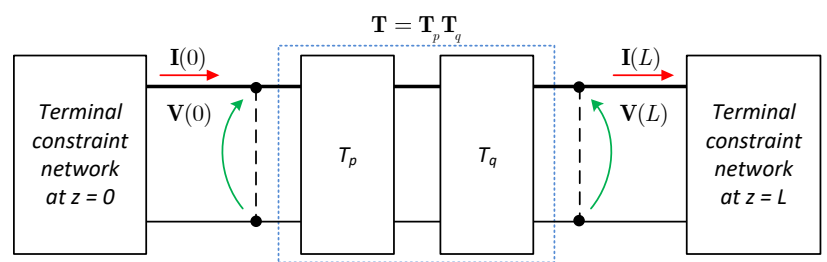

Fig. 2. $2 n$-port representation of a generic $(n+1)$-conductor NUTL obtained by cascading two $T$-parameter matrices and including terminal sections.

Recalling the definition of forward and reflected waves, and enforcing the aforementioned port constraints, the $n \times 1$ vectors collecting the currents at the left and right side of the TL under analysis are cast as

$$
\begin{gathered}
\mathbf{I}(0)=\left[\mathbf{n}_{1}^{-1}\left(\mathbf{Z}_{\mathrm{S}}-Z_{c} \mathbf{1}_{n}\right)-\mathbf{n}_{2}^{-1}\left(\mathbf{Z}_{\mathrm{S}}+Z_{c} \mathbf{1}_{n}\right)\right]^{-1} \\
\cdot\left(\mathbf{n}_{1}^{-1} \mathbf{k}_{1}-\mathbf{n}_{2}^{-1} \mathbf{k}_{2}\right) \\
\mathbf{I}(L)=\mathbf{n}_{1}^{-1} \mathbf{k}_{1}-\mathbf{n}_{1}^{-1}\left(\mathbf{Z}_{\mathrm{S}}-Z_{c} \mathbf{1}_{n}\right) \mathbf{I}(0)
\end{gathered}
$$

where

$$
\begin{gathered}
\left\{\begin{aligned}
\mathbf{n}_{1}= & \mathbf{T}_{\mathrm{L}, \mathrm{L}}\left(\mathbf{Z}_{\mathrm{L}}+Z_{c} \mathbf{1}_{n}\right)+\mathbf{T}_{\mathrm{L}, \mathrm{R}}\left(\mathbf{Z}_{\mathrm{L}}-Z_{c} \mathbf{1}_{n}\right) \\
\mathbf{n}_{2}= & \mathbf{T}_{\mathrm{R}, \mathrm{L}}\left(\mathbf{Z}_{\mathrm{L}}+Z_{c} \mathbf{1}_{n}\right)+\mathbf{T}_{\mathrm{R}, \mathrm{R}}\left(\mathbf{Z}_{\mathrm{L}}-Z_{c} \mathbf{1}_{n}\right)
\end{aligned}\right. \\
\left\{\begin{array}{l}
\mathbf{k}_{1}=\mathbf{V}_{\mathrm{S}}-\left(\mathbf{T}_{\mathrm{L}, \mathrm{L}}+\mathbf{T}_{\mathrm{L}, \mathrm{R}}\right) \mathbf{V}_{\mathrm{L}} \\
\mathbf{k}_{2}=\mathbf{V}_{\mathrm{S}}-\left(\mathbf{T}_{\mathrm{R}, \mathrm{L}}+\mathbf{T}_{\mathrm{R}, \mathrm{R}}\right) \mathbf{V}_{\mathrm{L}}
\end{array}\right.
\end{gathered}
$$

and $\mathbf{1}_{n}$ denotes the $n \times n$ identity matrix. The corresponding voltages are then obtained by substituting (13) in the equivalent Thevenin representation.

\section{ApPlication to TABbed Microstrip Lines}

In this section, the proposed approach is applied to differential microstrip lines with a surface tab-routing topology [10], [11], [18]. This design strategy was recently proposed by Intel Corporation to improve double data-rate performance, with the twofold objective to mitigate crosstalk with nearby traces as well as to achieve better impedance control, thus allowing longer and more compressed trace routing [10]. In this work, two examples of tabbed microstrip lines are considered. The first example deals with an interdigital tabbed microstrip line, the second involves a facing-tabbed microstrip line.

The accuracy of the advocated approach is proven by comparison of the obtained predictions versus the results of full-wave simulations. Moreover, the computational effectiveness will be investigated by comparison versus the original implementation of the perturbation technique [3], [4] as well as versus the traditional UCS method.

\section{A. Trapezoidal Interdigital Tabbed Microstrip Lines}

As a first test case, a pair of interdigital tabbed traces [10], consisting of interleaving trapezoidal tabs, is considered. Figs. 3 and 4 show the longitudinal and cross-sectional layout of the microstrip line under analysis, and demarcate pertinent geometrical and material parameters, whose values (if not reported in Fig. 3) are collected in Table I. From these parameters, the actual trace widths $w_{1}(z), w_{2}(z)$ and separation $s(z)$ in Fig. 4 are cast as function of the longitudinal coordinate $z$ by simple algebra. For voltage/current prediction at the microstrips' terminals, the four single-ended ports shown in Fig. 3 are identified, and the terminal conditions in Fig. 5 enforced.

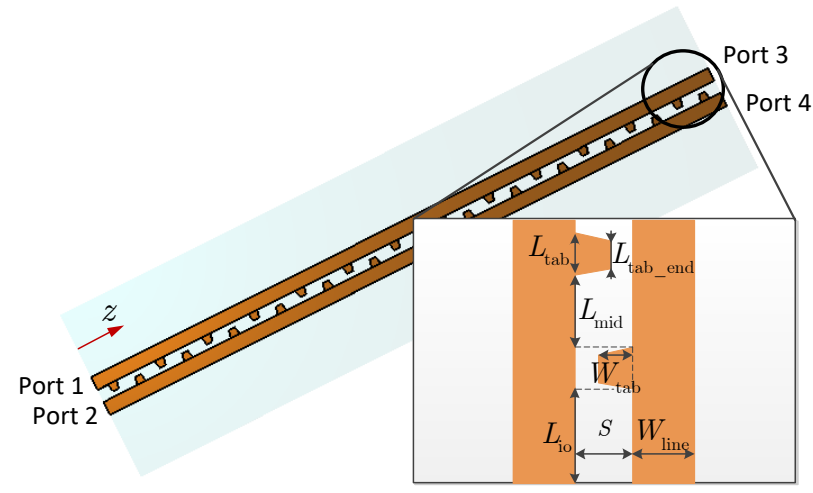

Fig. 3. Top view of the pair of trapezoidal interdigital-tabbed traces under analysis, indicating the terminal ports as well as pertinent geometrical parameters (see Table I for numerical values).

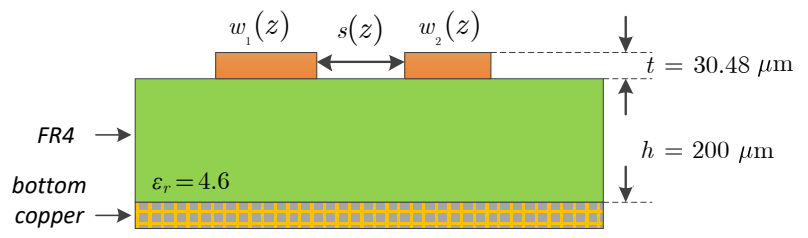

Fig. 4. Cross-sectional view of the microstrip lines in Fig. 3, indicating the geometrical dimensions and material characteristics. Trace widths $w_{1}(z)$, $w_{2}(z)$ and separation $s(z)$ are varying as function of the longitudinal coordinate $z$.

To calculate the voltages induced at the far-end (FE) terminals (i.e., ports 3 and 4 in Fig. 3) by the proposed method, the basic non-repetitive section labelled $\alpha$ in Fig. 6(a) is identified. 


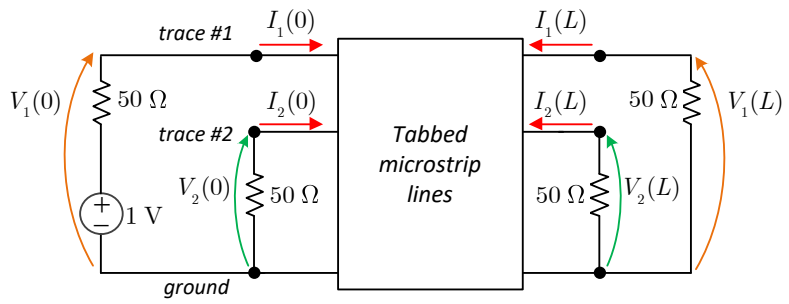

Fig. 5. Terminal conditions enforced at the single-ended ports of the microstrip lines in Fig. 3.

TABLE I

INTERDIGITAL-TABBED MICROSTRIP (FIG. 3): GEOMETRICAL PARAMETERS

\begin{tabular}{cc}
\hline \hline Parameter & Value \\
\hline Length & $19.3748 \mathrm{~mm}$ \\
$L_{\text {tab }}$ & $279.4 \mu \mathrm{m}$ \\
$L_{\text {tab_end }}$ & $177.8 \mu \mathrm{m}$ \\
$L_{\text {mid }}$ & $458 \mu \mathrm{m}$ \\
$L_{\text {io }}$ & $330.2 \mu \mathrm{m}$ \\
$W_{\text {line }}$ & $400 \mu \mathrm{m}$ \\
$W_{\text {tab }}$ & $152.4 \mu \mathrm{m}$ \\
$S$ & $380 \mu \mathrm{m}$ \\
\hline \hline
\end{tabular}

For such a basic cell, the pertinent p.u.l. parameters were numerically evaluated at $250 z$ positions along the section length. The entries of the corresponding $T$-parameter matrix $\mathbf{T}_{\alpha}$ are then evaluated column-by-column by the proposed perturbation technique as described in Section III where, now, $Z_{C}=50 \Omega$. Once matrix $\mathbf{T}_{\alpha}$ is known, the matrix $\mathbf{T}_{\beta}$ corresponding to the adjacent sub-section $\beta$ in Fig. 6(a) is obtained by introducing a suitable permutation matrix $\mathbf{T}_{t}$, i.e.,

$$
\mathbf{T}_{t}=\left[\begin{array}{llll}
0 & 1 & 0 & 0 \\
1 & 0 & 0 & 0 \\
0 & 0 & 0 & 1 \\
0 & 0 & 1 & 0
\end{array}\right]
$$

such that

$$
\mathbf{T}_{\beta}=\mathbf{T}_{t} \mathbf{T}_{\alpha} \mathbf{T}_{t}
$$

As shown in Fig. 6(a), the $T$-parameter matrix associated with entire NUTL network is obtained by cascading 13 repetitive cells characterized by $\mathbf{T}_{\alpha \beta}=\mathbf{T}_{\alpha} \mathbf{T}_{\beta}$ and by also including two uniform line sections at the ends.

Fig. 7 shows the comparison between the FE voltages predicted by the proposed approach versus those obtained by full-wave simulations with CST [19] (reference solution) and by the conventional UCS method. The latter required a line discretization into 4,000 uniform sections in order to achieve an accurate prediction. Apart from the slight deviation observed at low frequency (mainly to be ascribed to inherent limitations of the time-domain solver of the numerical software, in particular to the insufficient damping of the low-frequency fields by the absorbing boundaries), the good agreement among all curves proves the accuracy of the proposed TL-based approach despite the strong non-uniformity

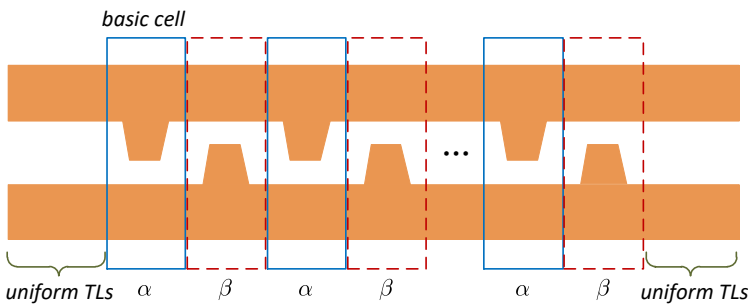

(a)

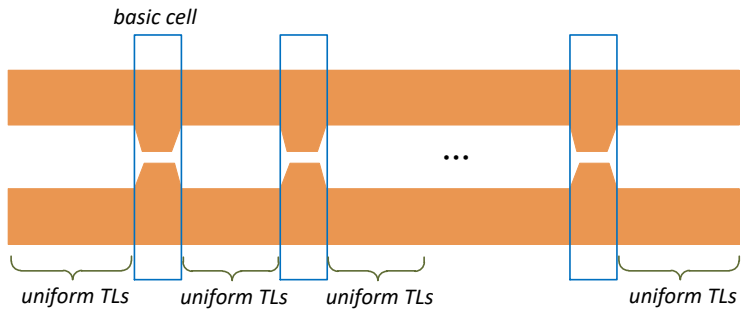

(b)

Fig. 6. Segmentation of tabbed microstrips into repetitive cells: (a) Interdigital tabbed lines; and (b) facing-tabbed lines.

affecting the structure under analysis. For completeness, it is worth mentioning that the accuracy of both TL-based methods decreases when the tab distance decreases (either due to an increase of $W_{t a b}$ or a descrease of $L_{m i d}$ ). This is due to nonnegligible capacitive coupling occurring between nearby tabs. These 3-D effects are not accounted for in TL theory, which assumes transverse electro-magnetic (TEM) propagation only. The recently-proposed tab coupling fringing capacitance compensation technique [18] might be invoked to further improve the prediction accuracy.

In this paper, the Electrostatics (es) interface in COMSOL Multiphysics [20] is utilized for numerical evaluation of the p.u.l. parameters. The parameter sweep and data extraction are automated by using the COMSOL APIs provided by LiveLink for MATLAB functionality embedded in COMSOL. In the special case of interdigital tabbed lines, the tab coupling fringing capacitance effect was included, by compensating the mutual capacitance $C_{m}$ as proposed in [18], and by accordingly modifying the entries of the capacitance matrices obtained by COMSOL.

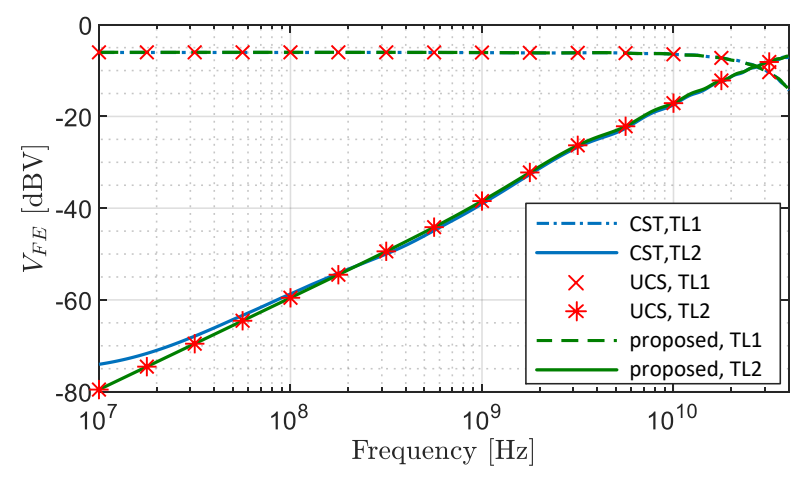

Fig. 7. Interdigital tabbed microstrip: Voltages at the FE terminals predicted by the full-wave solver (blue), the UCS method (red), and the proposed technique (green). TL1, TL2 denote traces 1 and 2, respectively. 
TABLE II

FACING-TABBED MICROSTRIP (FIG. 8): GEOMETRICAL PARAMETERS

\begin{tabular}{cc}
\hline \hline Parameter & Value \\
\hline Length & $18.6374 \mathrm{~mm}$ \\
$L_{\text {tab }}$ & $279.4 \mu \mathrm{m}$ \\
$L_{\text {tab_end }}$ & $177.8 \mu \mathrm{m}$ \\
$L_{\text {mid }}$ & $1195.4 \mu \mathrm{m}$ \\
$L_{i o}$ & $330.2 \mu \mathrm{m}$ \\
$W_{\text {line }}$ & $400 \mu \mathrm{m}$ \\
$W_{\text {tab }}$ & $101.6 \mu \mathrm{m}$ \\
$S$ & $380 \mu \mathrm{m}$ \\
\hline \hline
\end{tabular}

\section{B. Trapezoidal Facing-Tabbed Microstrip Lines}

As a second application example, the proposed technique is exploited to predict the voltages induced at the (FE) terminations of a trapezoidal facing-tabbed microstrip line [11], terminated as shown in Fig. 5. The line's top view, indicating its pertinent geometrical parameters whose numerical values are listed in Table II, is shown in Fig. 8. The geometrical and material parameters of the line's cross-section are the same as in Fig. 4, where $w_{1}(z), w_{2}(z)$, and $s(z)$ vary as function of the longitudinal coordinate $z$.

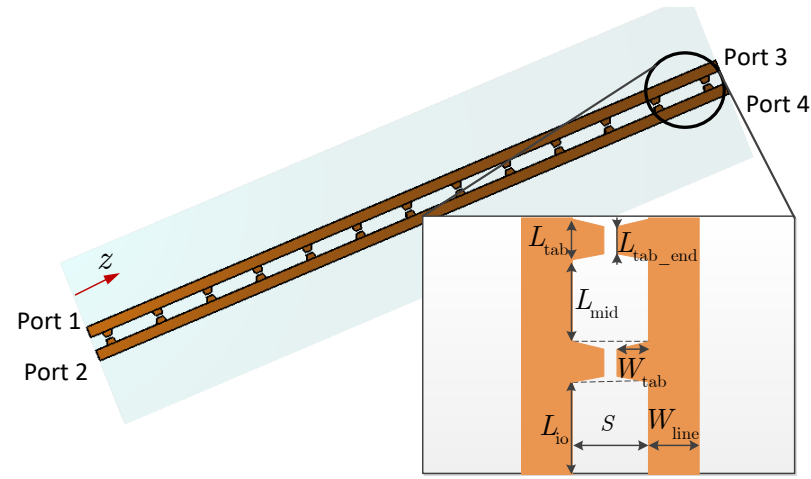

Fig. 8. Top view of the pair of facing-tabbed traces under analysis, indicating terminal ports as well as pertinent geometrical parameters (see Table II for numerical values).

For an efficient line solution, exploiting the proposed technique, two basic cells were identified as shown in Fig. 6(b). To evaluate the $S$-parameter matrix associated with the basic cell, encompassing the facing-tabbed section, the pertinent p.u.l. parameters were numerically evaluated at 150 line positions along $L_{t a b}$. The obtained matrix was then cascaded with the one associated with the second basic cell, corresponding to a uniform TL section. Enforcing the terminal constraints in Fig. 5 at the ports of the cascaded connection of the $T$-matrices associated with all basic cells yields the predictions (viz., the FE voltages induced at ports 3 and 4 in Fig. 8) shown in Fig. 9, which satisfactory agree with those obtained by the full-wave solver (blue curves) as well as with the UCS method (red markers). As previously observed, the discrepancies observed at low frequencies are ascribed to limitations of the timedomain full-wave solver, rather than to inaccuracy of TL-based approaches.

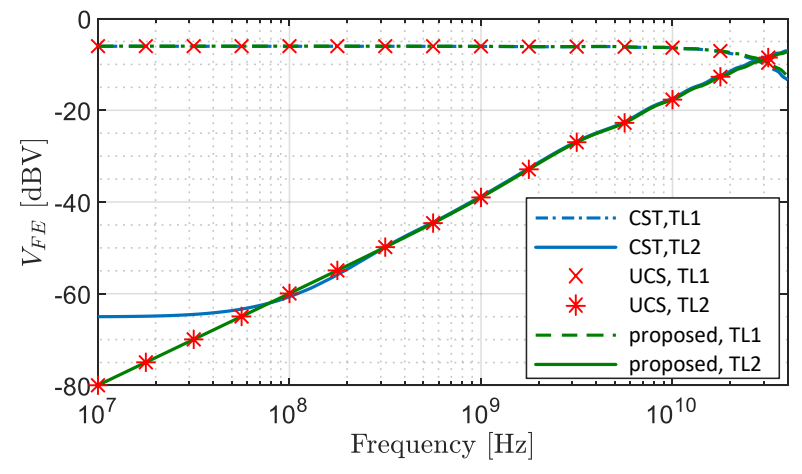

Fig. 9. Facing-tabbed microstrip: Voltages at the FE terminals predicted by the full-wave solver (blue), the UCS method (red), and the proposed technique (green). TL1, TL2 denote traces 1 and 2, respectively.

\section{Computational Efficiency}

In this section, the computational efficiency of the proposed technique is assessed by comparison with the original perturbation technique (which solves the entire line as a whole), as well as with the results obtained by the UCS method. This latter method comes in two flavours: (i) solving the line as a whole on the one hand, and (ii) solving a single cell and subsequent cascading of the obtained matrices, similar to the proposed method. The comparison of required computational times is summarized in Table III. For the perturbative solution, a threshold $\delta_{t h}=1 \%$ is set. For the UCS method, the number of uniform sections was chosen equal to the number of intermediate cross-sections at which the p.u.l. parameters were evaluated.

Simulations were performed on a standard desktop PC with an Intel(R) Core(TM) i5-7400 CPU running at $3.0 \mathrm{GHz}$ and $16 \mathrm{~GB}$ of RAM, without taking advantage of parallel computing (an option that is, however, now made available by the proposed solution technique). The FE voltages were calculated at 3603 frequency points (1000 logarithmicallyspaced points per decade) in the interval from $10 \mathrm{MHz}$ up to $40 \mathrm{GHz}$. In addition to standard single-thread computation, the Parallel Computing Toolbox available in MATLAB was used to further accelerate the solution.

As expected, the two solution schemes taking advantage of periodicity of the structures under analysis are definitely faster than those solving the entire line as a whole. Among these, the proposed solution approach is proven to be much more computationally-effective than the UCS solution. In the case of interdigital tabbed lines, this is owing to the high degree of nonuniformity. Although the facing-tabbed microstrip includes several uniform TL sections, whose computational time is nearly negligible, a considerable speed-up is still achieved. With the further aid of parallel-computing, the calculation time of the proposed technique is reduced to approximately a quarter the time for the single-thread (slightly larger due to file access, thread dispatch, etc.), providing even more significant acceleration with respect to the UCS method. 


\section{APPLiCATION TO CM SUPPRESSION In DifFERENTIAL MICROSTRIP LINES}

In this section, the proposed technique is applied to predict the performance of microstrip lines with common-mode $(\mathrm{CM})$ suppression based on electromagnetic band-gaps (EBGs). This technology was recently proposed in [12], [13], and, with respect to other solutions, it offers the advantage that the ground plane is left unaltered, yet assuring effective CM mitigation at specific frequencies.

The microstrip layout is shown in Fig. 10. This is the exact same layout as the one proposed in [12], which is characterized by four cells with length $l=2.38 \mathrm{~cm}$, and weighting factor $K_{1}=0.305 \mathrm{~cm}$. The microstrip substrate (Rogers RO3010) has dielectric constant $\epsilon_{r}=10.2$ and thickness $h=1.27 \mathrm{~mm}$. Equation (6) in [12] can be exploited to calculate the varying $\mathrm{CM}$ impedance $Z_{C M}$ along the longitudinal $z$-axis, with terminal value $Z_{C M}(0)=Z_{C M}(L)=50 \Omega$, whereas the differential mode (DM) impedance is kept constant to the value $Z_{D M}(z)=50 \Omega$. For further details about the design, we refer the interested reader to [12]. The microstrip geometry is imported into the 3D full-wave solver ADS [21], and the mixed-mode $\mathrm{S}$-parameters obtained by numerical simulations are taken as reference quantities.

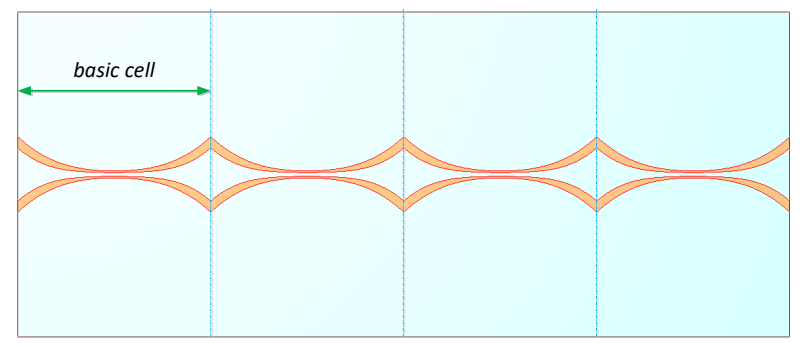

Fig. 10. Top view of the CM-suppressed differential microstrip under analysis [12].

For our TL-based solution, the p.u.l parameters of the basic cell (shown in Fig. 10) were numerically evaluated, and the corresponding $S$-parameter matrix was then calculated by the proposed perturbation technique. Cascading the obtained $T$-matrices, and subsequently converting the obtained result into a mixed-mode $S$-parameter description, yields the solid curves in Fig. 11. The comparison versus the same quantities computed by full-wave simulation (dashed curves) reveals a satisfactory agreement, especially for the mixed-mode transmission coefficients in the frequency interval around 2-2.5 $\mathrm{GHz}$, where the filter is expected to provide the largest $\mathrm{CM}$ suppression. Slight discrepancies are mainly observed in the DM reflection coefficients $S_{11}^{d d}$, which ideally should be null in case of perfect DM matching.

In terms of computational time, the comparison in Table III confirms that the proposed technique outperforms the other TL-based methods, yet preserving the same prediction accuracy. Indeed, the results obtained by the other TL-based techniques were omitted in Fig 11, since they were perfectly overlapping with the curves obtained by the proposed technique.
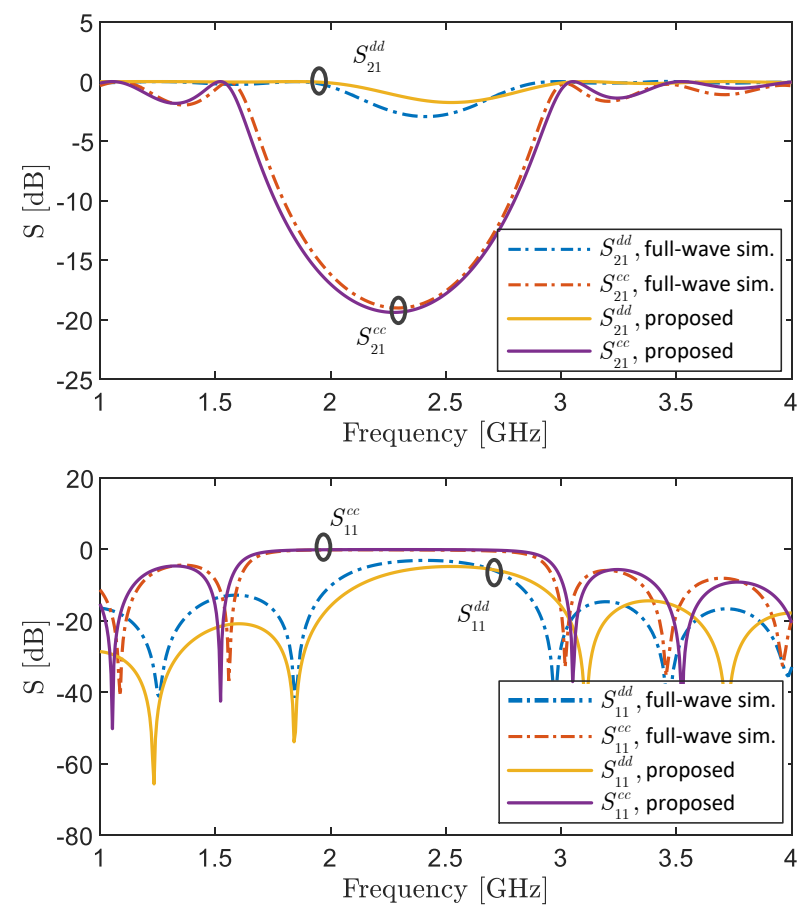

Fig. 11. Comparison of $S$-parameters for the differential microstrip line structure of Fig. 10. Dashed lines are full-wave simulation results, and solid lines stem from the TL-based solution techniques, which are perfectly overlapping with one another.

TABLE III

Computational Time

\begin{tabular}{cccc}
\hline & interdigital tabbed & facing tabbed & CM-suppression \\
\hline UCS - whole line & $95.4 \mathrm{~s}$ & $57.7 \mathrm{~s}$ & $256.6 \mathrm{~s}$ \\
UCS - cascaded & $63.2 \mathrm{~s}$ & $33.5 \mathrm{~s}$ & $134.9 \mathrm{~s}$ \\
standard PT - whole line & $61.3 \mathrm{~s}$ & $35.9 \mathrm{~s}$ & $208.7 \mathrm{~s}$ \\
proposed PT - sequential & $48.3 \mathrm{~s}$ & $31.8 \mathrm{~s}$ & $108.8 \mathrm{~s}$ \\
proposed PT - parallel & $13.3 \mathrm{~s}$ & $8.0 \mathrm{~s}$ & $27.5 \mathrm{~s}$ \\
\hline \hline
\end{tabular}

\section{CONCLUSION}

In this manuscript, a novel procedure for the solution of NUTLs has been presented. The procedure makes use of the perturbation technique in [4] as key ingredient, but, in contrast to its original implementation, it provides a representation of the NUTL under analysis in terms of a $2 n$-port network. This is achieved by calculating the entries of the corresponding $S$-parameter matrix column-by-column, by solution of $2 n$ sub-problems. The increase in computational burden can be mitigated by exploiting repetitive properties of the structure under analysis or parallel computing, and it is anyway offset by the higher flexibility offered by the obtained $2 n$-port representation, which can be cascaded with other networks and/or (re)used in combination with different sets of terminal loads.

The proposed procedure was proven to be particularly effective in the modeling of differential lines exhibiting periodic geometries. Namely, for these types of NUTLs, the advantage is twofold. First, the number of sub-problems to be solved is limited to $2 n=4$. Second, the solution can be conveniently obtained by identifying a basic cell, characterising it in terms 
of its $S / T$-parameters, and representing the entire line as a cascade connection of these basic cell.

Three application examples were presented, which involve differential microstrips with non-uniform but periodic trace routing. For these examples, predictions obtained by the proposed procedure have been firstly compared versus those provided by full-wave simulations to investigate the effectiveness and possible limitations of solution approaches based on TL theory (i.e., on the assumption of transverse electromagnetic wave propagation) in the presence of significant line nonuniformity. Afterwards, the computational efficiency of the proposed procedure was assessed by comparison versus the original implementation of the perturbation technique in [4] and the standard solution approach based on line subdivision into uniform cascaded sections (UCS). Even without parallel computing, it was proven that resorting to the proposed procedure for the solution of periodic NUTLs offers computational advantages not only with respect to the original perturbation technique, which requires solving the entire line as a whole, but also with respect to the UCS method, which can benefits from the possibility to solve periodic NUTLs as the a cascade of basic cells, similar to the advocated procedure.

The achieved computational efficiently, which can be further improved by parallel computing, makes the proposed procedure definitely attractive to support and improve the design of high-speed interconnects. As a matter of fact, resorting to differential microstrips involving periodic trace routing is a design solution recently gaining increasing attention due to the possibility to reduce interference. This is the case for the specific realisations analyzed in the manuscript, which were recently proposed with the objective to reduce crosstalk (see the tabbed lines in Section IV) and CM-noise (see the EBGbased microstrip in Section V).

\section{REFERENCES}

[1] C. R. Paul, Analysis of multiconductor transmission lines. John Wiley \& Sons, 2008

[2] J. F. Mao and Z. F. Li, "Analysis of the time response of nonuniform multiconductor transmission lines with a method of equivalent cascaded network chain," IEEE Trans. Microw. Theory Techn., vol. 40, no. 5, pp. 948-954, 1992.

[3] M. Chernobryvko, D. Vande Ginste, and D. De Zutter, "A two-step perturbation technique for nonuniform single and differential lines," IEEE Trans. Microw. Theory Techn., vol. 61, no. 5, pp. 1758-1767, 2013.

[4] P. Manfredi, D. De Zutter, and D. Vande Ginste, "Analysis of nonuniform transmission lines with an iterative and adaptive perturbation technique," IEEE Trans. Electromagn. Compat., vol. 58, no. 3, pp. 859-867, 2016.

[5] X. Wu, F. Grassi, P. Manfredi, and D. Vande Ginste, "Perturbative analysis of differential-to-common mode conversion in asymmetric nonuniform interconnects," IEEE Trans. Electromagn. Compat., vol. 60, no. 1 , pp. 7-15, 2018.

[6] P. Manfredi, X. Wu, F. Grassi, D. Vande Ginste, and S. A. Pignari, "A perturbative approach to predict eye diagram degradation in differential interconnects subject to asymmetry and nonuniformity," in Proc. IEEE 21 st Workshop Signal Power Integrity. IEEE, May 2017, pp. 1-4.

[7] X. Wu, P. Manfredi, D. V. Ginste, and F. Grassi, "A Hybrid PerturbativeStochastic Galerkin Method for the Variability Analysis of Nonuniform Transmission Lines," IEEE Trans. Electromagn. Compat., to be published, doi: 10.1109/TEMC.2019.2922407.

[8] A. R. Mallahzadeh, A. Ghasemi, S. Akhlaghi, B. Rahmati, and R. Bayderkhani, "Crosstalk reduction using step shaped transmission line," Progress In Electromagnetics Research C, vol. 12, pp. 139-148, 2010.
[9] S.-K. Koo, H. Lee, and Y.-B. Park, "Crosstalk Reduction Effect of Asymmetric Stub Loaded Lines," J. Electromagn. Waves Appl., vol. 25, pp. 1156-1167, 2011.

[10] R. K. Kunze, Y. Chu, Z. Yu, S. K. Chhay, M. Lai, and Y. Zhu, "Crosstalk mitigation and impedance management using tabbed lines," Intel white paper, 2015.

[11] M. Baldwin and V. Di Lello, "New techniques to address layout challenges of high-speed signal routing," Cadence, PCB West, Tech. Rep., 2016.

[12] P. Vélez, J. Bonache, and F. Martín, "Differential microstrip lines with common-mode suppression based on electromagnetic band-gaps (EBGs)," IEEE Antennas Wireless Propag. Lett., vol. 14, pp. 40-43, 2015.

[13] P. Vélez, M. Valero, L. Su, J. Naqui, J. Mata-Contreras, J. Bonache, and F. Martín, "Enhancing common-mode suppression in microstrip differential lines by means of chirped and multi-tuned electromagnetic bandgaps," Microw. Opt. Technol. Lett., vol. 58, no. 2, pp. 328-332, 2016.

[14] H. Takeda, K. Iokibe, Y. Toyota, W. Koh, and W. Chang, "Crosstalk reduction by introducing periodic structure into dense differential pairs," in Proc. 2017 Asia-Pac. Int. Symp. Electromagn. Compat., Seoul, South Korea, 2017, p. 353.

[15] C. Wang, H. Takeda, K. Iokibe, and Y. Toyota, "Reduction mechanism of differential-mode crosstalk between adjacent differential pairs with periodic structure," in 2019 Asia-Pacific Symposium on Electromagnetic Compatibility (APEMC), 2019.

[16] D. M. Pozar, Microwave engineering. Wiley, 2012.

[17] T. Reveyrand, "Multiport conversions between S, Z, Y, h, ABCD, and T parameters," in Proc. IEEE Integr. Nonlinear Microw. Millim.-Wave Circuits Workshop. IEEE, Jul. 2018, pp. 1-3.

[18] W. Jiang, X.-D. Cai, B. Sen, and G. Wang, "Equation-based solutions to coupled, asymmetrical, lossy, and nonuniform microstrip lines for tab-routing applications," IEEE Trans. Electromagn. Compat., 2018.

[19] Dassault Systèmes, "CST Studio Suite: electromagnetic field simulation software," https://www.3ds.com/products-services/simulia/products/cststudio-suite/, (Accessed Aug 08, 2019).

[20] COMSOL INC., "COMSOL Multiphysics Documentation," https://www.comsol.com/documentation, (Accessed Dec 08, 2019).

[21] Keysight Technologies, "ADS Product Documentation," http://edadocs.software.keysight.com/display/ads2017update1/Home/, (Accessed July 15, 2019). 